\title{
Produção de mudas de amora preta com substrato a base de caule decomposto de babaçu
}

$\mathrm{Na}$ formação de mudas de amoreira preta-preta (Rubus spp.) é fundamental a combinação de substratos para se obter mudas de qualidade. Com isso, avaliou-se a influência do caule decomposto de babaçu (CDB) na formação de mudas de amoreira preta-preta. Conduzido em casa de vegetação com delineamento inteiramente casualizado com 6 tratamentos e 4 repetições, sendo: S1 - 0\% CDB; S2 - 20\% CDB + 80\% S; S3- 40\% CDB + 60\% S; S4 - 60\% CDB + 40\% S; S5 - 80\% CDB + 20\% S; S6 - 100\% CDB. Os resultados foram submetidos à análise de variância pelo teste "F" e as médias comparadas pelo Teste de Tukey a $5 \%$ de significância. Os tratamentos testados não apresentaram diferença significativa nas variáveis número de folhas (NF), comprimento do maior broto (CMB), diâmetro de maior broto (DMB) e comprimento radicular (CR), revelando independência das proporções. Para as demais variáveis, área foliar (AF), volume radicular (VR) e as massas fresca da parte aérea (MFPA) e massa fresca da raiz (MFR), os substratos produzidos com as maiores concentrações de caule decomposto de babaçu, favoreceram as variáveis destacadas. As concentrações de teores totais de nutrientes observado na análise química e as características física do substrato, devem ter proporcionado o desenvolvimento nas variáveis mencionadas. Embora a composição dos substratos testados em combinação com solo não tenha sido eficiente para as principais variáveis, o substrato contendo $100 \%$ de babaçu de um modo geral, mostrou melhor eficiência na produção de mudas de amoreira-preta.

Palavras-chave: Rubus ssp.; Attalea speciosa Mart.; Frutíferas.

\section{Production of blackberry seedlings with stem base substrate decorated Babaçu}

In the formation of black-black mulberry seedlings (Rubus spp.) It is essential to combine substrates to obtain quality seedlings. Thus, the influence of the decomposed babassu stem (DBS) on the formation of black-black mulberry seedlings was evaluated. Conducted in a greenhouse with a completely randomized design with 6 treatments and 4 repetitions, being: S1 - 0\% DBS; S2 - 20\% DBS + 80\% S; S3- 40\% DBS + 60\% S; S4 - 60\% DBS + 40\% S; S5 - 80\% DBS + 20\% S; S6 $100 \%$ DBS. The results were subjected to analysis of variance by the " $F$ " test and the means compared by the Tukey test at $5 \%$ significance. The treatments tested showed no significant difference in the variables number of leaves (NL), length of the largest shoot (LLS), diameter of largest shoot (DLS) and root length (RL), revealing independence of proportions. For the other variables, leaf area (LA), root volume (RV) and the fresh mass of the aerial part (FMAP) and fresh mass of the root (FMR), the substrates produced with the highest concentrations of decomposed babassu stem, favored the highlighted variables. The concentrations of total nutrient contents observed in the chemical analysis and the physical characteristics of the substrate, must have provided the development in the mentioned variables. Although the composition of the tested substrates in combination with soil was not efficient for the main variables, the substrate containing $100 \%$ babassu in general, showed better efficiency in the production of blackberry seedlings.

Keywords: Rubus ssp.; Attalea speciosa Mart.; Fruit.

Topic: Uso Sustentável da Biodiversidade

Received: 09/04/2021

Approved: 08/05/2021

Reviewed anonymously in the process of blind peer.

Kleber Veras Cordeiro (iD

Universidade Federal do Maranhão, Brasil http://lattes.cnpq.br/7585883012639032 http://orcid.org/0000-0003-0149-8819 kleberverascordeiro@hotmail.com

Paula Sara Teixeira de Oliveira (iD) Universidade Federal do Maranhão, Brasil http://lattes.cnpq.br/3559574180065279 http://orcid.org/0000-0001-8968-7061 paulasara1997@gmail.com

Amalia Santos da Silva Veras (iD) Universidade Federal do Maranhão, Brasil http://lattes.cnpq.br/2440383630370636 http://orcid.org/0000-0002-2553-635X amaliasantos565@gmail.com
Vanessa Brito Barroso (iD

Universidade Federal do Maranhão, Brasil http://lattes.cnpq.br/6848925247182712 http://orcid.org/0000-0002-6675-5110 vanessaflick@hotmail.com

Maurivan Barbosa Pachêco (ic

Universidade Federal do Maranhão, Brasil http://lattes.cnpq.br/2593810903242525 http://orcid.org/0000-0002-6146-9932 ivanbpacheco@hotmail.com

João Pedro Santos Cardoso (iD

Universidade Federal do Maranhão, Brasil http://lattes.cnpq.br/1057085207810749 http://orcid.org/0000-0003-4465-8712 joaopedrocardos020@hotmail.com
Raissa Rachel Salustriano da Silva Matos (if Universidade Federal do Maranhão, Brasil http://lattes.cnpq.br/0720581765268326 http://orcid.org/0000-0002-8908-2297 raissasalustriano@yahoo.com.br

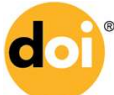

DOI: 10.6008/CBPC2179-6858.2021.005.0016
Referencing this:

CORDEIRO, K. V.; OLIVEIRA, P. S. T.; VERAS, A. S. S.; BARROSO, V. B.; PACHÊCO, M. B.; CARDOSO, J. P. S.; MATOS, R. R. S. S.. Produção de mudas de amora preta com substrato a base de caule decomposto de babaçu. Revista Ibero Americana de Ciências Ambientais, v.12, n.5, p.180-187, 2021. DOI: http://doi.org/10.6008/CBPC2179$\underline{6858.2021 .005 .0016}$ 


\section{INTRODUÇÃO}

O cultivo de amoreira-preta possui baixo custo de manutenção, sendo considerada uma boa opção para diversificação de áreas de produção frutícola, com rápido retorno dos investimentos a partir do segundo ano de implantação da cultura (SCHIEHL et al., 2020). Com a possibilidade de diversificação de produtos, a amoreira preta (Rubus spp.) é uma fruta promissora para aproveitamento total, inclusive como ornamental por suas relevantes características nas folhas, flor e frutos (BICCA et al., 2020).

No mercado de frutas exóticas, a amora preta apresenta destaque em função de possuir significativas quantidades de compostos de interesse fármaco. Elas são ricas em vitamina $C$ e contêm em torno de $10 \%$ de carboidratos, $85 \%$ de água, elevado conteúdo de minerais, vitaminas do complexo A e B, além de ser fonte de compostos funcionais, como ácido elágico e antocianinas.

Com o aumento de áreas cultivadas com essa frutífera, a avaliação de cultivares de amoreira na região tropical é uma alternativa agrícola para produtores. Na propagação de amoreira - preta, podem ser utilizadas estacas herbáceas, lenhosas e de raízes, além de rebentos e da cultura de tecidos (ANTUNES et al., 2004). O processo de formação de raízes em estacas está relacionado ao substrato, que exerce influência na qualidade de formação das raízes (LONE et al., 2010). Segundo Sá et al. (2013) respostas favoráveis ao desenvolvimento de mudas frutíferas, vem sendo constantemente reportadas com a utilização de substratos alternativos advindos de componentes orgânicos e que podem reduzir custos na produção de mudas.

O enraizamento de estacas depende da espécie, do tipo de estaca, da época, do sistema de propagação e do substrato (Le Bellec et al., 2006). Sendo este um insumo básico, como suporte físico que permite a retenção de água, o arejamento e agregação do sistema radicular, além de disponibilizar recursos, como nutrientes essenciais, boa capacidade de troca catiônica, ausência de agentes patogênicos, pH entre 5,5 e 6 e baixa salinidade (Farias et al., 2012).

É fundamental a seleção de materiais que possibilite a retenção de água suficiente para evitar que as estacas sequem e que este material possibilite fornecimento de oxigênio, e possua espeço poroso necessária à iniciação e o desenvolvimento radícula (BICCA et al., 2020).

Uma vez que, a adoção do substrato adequado influencia diretamente na qualidade das mudas, sendo as características físicas e químicas determinantes, afetando tanto o crescimento quanto a produção (Maggioni et al., 2014). Associado a isto, é importante conhecer a biodiversidade regional para buscar alternativas que venham a diminuir cada vez mais o custo da etapa de produção de mudas (COELHO et al., 2013).

A palmeira de babaçu (Attalea speciosa Mart.), considerada endêmica no Maranhão, ocupa boa parte do estado formando a chamada mata de cocais, quando em senescência e após queda sua estipe decomposta apresenta alto teor de matéria orgânica, pode ser uma alternativa de uso na produção de mudas de amora preta. Nesse contexto, o objetivo foi avaliar a eficiência do substrato caule decomposto de babaçu na formação de mudas de amoreira - preta pelo método de enraizamento de estacas. 


\section{METODOLOGIA}

A pesquisa foi conduzida na área experimental do Centro de Ciências Agrárias e Ambientais/Universidade Federal do Maranhão (CCAA/UFMA) - Campus IV, no município de Chapadinha MA ("0344'28,7" S; "4318'46" W e $107 \mathrm{~m}$ de altitude) em casa de vegetação. O clima nesta região é classificado como tropical úmido, com precipitação média anual de $1613 \mathrm{~mm}$ e temperatura média anual de 27,9 으 (PASSOS et al., 2016).

Foram coletados ramos semi-lenhosos no período da tarde, sendo todo o material botânico utilizado, procedente de árvores matrizes com bom estado nutricional e fitossanitário. As estacas foram preparadas com auxílio de tesoura de poda, com corte em bisel na base e acima da gema axilar. Foram preparadas estacas com $25 \mathrm{~cm}$ de comprimento e diâmetro $(0,8-1 \mathrm{~cm})$, sendo a superfície foliar reduzida totalmente visando homogeneizar a amostragem. O plantio foi realizado em sacos de poliestireno contendo diferentes proporções de substratos a base de caule decomposto de babaçu. $\mathrm{O}$ experimento foi conduzido em delineamento inteiramente casualizado, com 6 tratamentos e quatro repetições, com cada parcela contendo 16 mudas, gerando um total de 384 mudas. Os tratamentos foram substratos formulados com $0 \%, 20 \%, 40 \%, 60 \%, 80 \%$ e $100 \%$ de caule decomposto de babaçu, acrescidos de solo (LAd).

Nas Tabelas 1 e 2 pode-se verificar a caracterização química e física dos substratos. E no solo que compõem os substratos com CDB, foi realizada análise granulométrica: 384 g areia grossa $/ \mathrm{kg} ; 336 \mathrm{~g}$ areia fina/kg; $112 \mathrm{~g}$ de silte/kg; $168 \mathrm{~g}$ de argila total $/ \mathrm{kg} ; 38 \mathrm{~g}$ de argila natural $/ \mathrm{kg}$; classificação textural Franco arenosa; e grau de floculação de $77 \mathrm{~g} / 100 \mathrm{~g}$.

Tabela 1: Valores de $\mathrm{pH}$, condutividade elétrica (CE) e teores totais de (N), fósforo (P), potássio ( $K)$, cálcio (Ca) e magnésio (Mg) e enxofre (S), do substrato comercial e dos substratos a base de caule decomposto de babaçu (CDB).

\begin{tabular}{|c|c|c|c|c|c|c|c|c|}
\hline \multirow[t]{2}{*}{ CDB \% } & \multirow[t]{2}{*}{$\mathrm{pH}$} & \multirow{2}{*}{$\begin{array}{l}\mathrm{CE} \\
\mathrm{dS} \mathrm{m}^{-1}\end{array}$} & \multirow{2}{*}{$\begin{array}{l}\mathrm{N} \\
\mathrm{g} \mathrm{kg}^{-1}\end{array}$} & \multirow{2}{*}{$\begin{array}{l}\mathrm{P} \\
\mathrm{mg} \mathrm{kg}^{-1}\end{array}$} & $\mathrm{~K}$ & $\mathrm{Ca}$ & $\mathrm{Mg}$ & \multirow[t]{2}{*}{$\mathrm{S}$} \\
\hline & & & & & \multicolumn{3}{|c|}{$\longrightarrow \mathrm{cmol}_{\mathrm{C} \mathrm{kg}} \mathrm{k}^{-1}$} & \\
\hline S1 & 5,06 & 0,10 & 0,63 & 13 & 0,07 & 0,80 & 0,30 & 1,05 \\
\hline S2 & 4,88 & 0,61 & 1,23 & 14 & 0,67 & 1,60 & 1,00 & 3,8 \\
\hline S3 & 5,11 & 1,36 & 1,46 & 13 & 1,82 & 3,20 & 1,70 & 7,6 \\
\hline S4 & 4,83 & 1,79 & 2,02 & 13 & 2,35 & 4,40 & 2,80 & 10,8 \\
\hline S5 & 5,16 & 3,00 & 3,47 & 27 & 6,17 & 10,90 & 4,60 & 24,6 \\
\hline S6 & 5,32 & 4,34 & 5,88 & 33 & 3,63 & 20,60 & 15,20 & 41,5 \\
\hline
\end{tabular}

S1 $=100 \%$ solo; $S 2=20 \%$ de CDB $+80 \%$ de Solo; S3= $40 \%$ de CDB + $60 \%$ de Solo; $S 4=60 \%$ de CDB + de $40 \%$ Solo; $\mathrm{S} 5=80 \%$ de CDB $+20 \%$ de Solo; e $\mathrm{S} 6=100 \%$ de CDB.

Foi realizado o estaqueamento à profundidade de $1 / 3$ do seu tamanho dispostas nos sacos de poliestireno e mantidas em casa de vegetação com uma irrigação diária. Ao final do experimento, 53 dias após a estaquia, foram avaliadas as seguintes variáveis: I - número de folhas (NF): obtido por contagem manual; II - área foliar (AF): aferida através do escaneamento das folhas e mensurada com auxílio do programa computacional Image $J^{\oplus}$;III - comprimento do maior broto (CMB): medido com uma régua melimetrada; IV - diâmetro do maior broto (DMB): obtido com paquímetro digital (Digimess'); V comprimento da maior raiz (CMR): medido com auxílio de uma régua graduada em milímetros; $\mathrm{VI}$ - volume radicular (VR): realizado por meio de medição do deslocamento da coluna de água em proveta graduada, segundo metodologia descrita por Basso (1999); VII - massa fresca da raiz (MFR) e VIII - massa fresca de parte área (MFPA): para obtê-las o material vegetal foi pesado em balança com precisão de $0,01 \mathrm{~g}$. 
Tabela 2: Densidade global (DG), densidade de partícula (DP) e porosidade (P) do substrato comercial e dos substratos a base de caule decomposto de babaçu (CDB).

\begin{tabular}{llll}
\hline \multirow{2}{*}{ Substratos } & \multicolumn{2}{l}{ Densidade $\left(\mathrm{g} / \mathrm{cm}^{3}\right)$} & Porosidade (\%) \\
\cline { 2 - 3 } & DG & DP & 34,43 \\
\hline S1 & 0,56 & 0,85 & 51,53 \\
S2 & 1,28 & 2,64 & 54,01 \\
S3 & 1,18 & 2,57 & 56,22 \\
S4 & 0,98 & 2,24 & 60,91 \\
S5 & 0,73 & 1,88 & 65,95 \\
S6 & 0,33 & 0,97 & \\
\hline
\end{tabular}

S1 $=100 \%$ solo; $S 2=20 \%$ de CDB $+80 \%$ de Solo; $S 3=40 \%$ de CDB $+60 \%$ de Solo; $S 4=60 \%$ de $C D B+$ de $40 \%$ Solo; $S 5=$ $80 \%$ de $\mathrm{CDB}+20 \%$ de Solo; e $\mathrm{S} 6=100 \%$ de $\mathrm{CDB}$.

Os dados foram submetidos à análise de variância pelo teste "F", para diagnóstico de efeito significativo, e os tratamentos comparados entre si pelo teste Tukey a $5 \%$ de probabilidade, através do programa Software Assistat ${ }^{\circ}$ V 7.7 (SILVA et al., 2016).

\section{RESULTADOS E DISCUSSÃO}

A análise de variância (Tabela 3) não revelou efeito significativo com os substratos compostos por caule decomposto de babaçu para as variáveis número de folhas, comprimento do maior broto, diâmetro de maior broto e comprimento radicular, mostrando que o desenvolvimento dessas variáveis foi independente das proporções do substrato utilizado. No entanto, houve efeito significativo para as variáveis área foliar, volume radicular e para as massas frescas da parte aérea e do sistema radicular.

Tabela 3: Análise de variância dos parâmetros avaliados em estacas de amora submetidas a diferentes concentrações de substrato caule decomposto de babaçu e solo.

\begin{tabular}{|c|c|c|c|c|c|c|c|c|}
\hline \multirow[b]{2}{*}{ FV } & \multicolumn{8}{|c|}{ Quadrados médios } \\
\hline & $N F$ & $\begin{array}{l}\text { CMB } \\
(\mathrm{cm})\end{array}$ & $\begin{array}{l}\text { DMB } \\
(\mathrm{mm})\end{array}$ & $\begin{array}{l}\text { CMR } \\
(\mathrm{cm})\end{array}$ & $\begin{array}{l}\mathrm{AF} \\
\left(\mathrm{cm}^{2}\right)\end{array}$ & $\begin{array}{l}\text { VR } \\
\left(\mathrm{cm}^{3}\right)\end{array}$ & MFR & MFPA \\
\hline Substratos & 9,77 & 11,58 & 0,14 & 5,45 & 53691,16 & 0,90 & 1,34 & 8,20 \\
\hline Resíduo & 3,32 & 7,98 & 0,06 & 10,28 & 6886,69 & 0,15 & 0,36 & 0,78 \\
\hline SIG & $*$ & Ns & ns & ns & $* *$ & $* *$ & $*$ & $* *$ \\
\hline $\mathrm{CV}(\%)$ & 23,29 & 21,60 & 10,46 & 19,29 & 22,29 & 18,20 & 25,78 & 25,38 \\
\hline
\end{tabular}

$\mathrm{CV}=$ coeficiente de variação. $^{* *}$ : significativo ao nível de $1 \%$ de probabilidade $(p<0,01) ;{ }^{*}:$ significativo ao nível de $5 \%$ de probabilidade $(p<0,05) ;{ }^{\text {ns: }}$ não significativo $(p>=0,05)$.

Em relação ao número de folhas por planta, embora o resultado não tenha sido significativo, houve um aumento nas medias, à medida que as concentrações de CDB aumentavam, fazendo com que as estacas apresentassem uma média de 10,96 folhas com a $56(100 \%)$, enquanto que para esta mesma variável com o substrato $\mathrm{S} 1(0 \% \mathrm{CDB})$ a média foi de 7,38 . Para o comprimento do maior broto não foi diferente, os resultados demonstraram que à medida que aumentava a concentração de CDB, promovia um aumento na média de crescimento das estacas de amora (Figura 1).
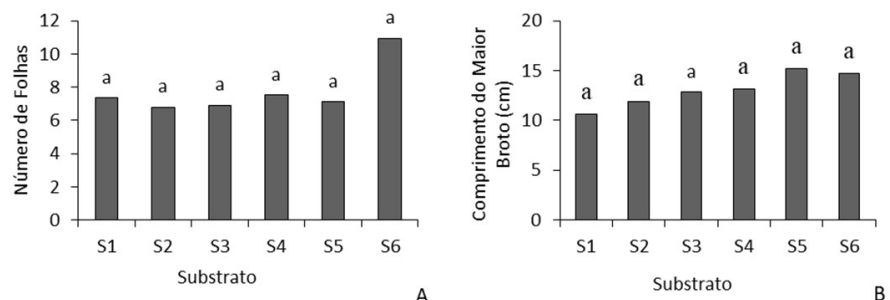

Figura 1: Número de folhas (A) e comprimento do maior broto (B) de mudas de amora preta com substrato a base de caule decomposto de babaçu acrescido de solo. 
Para o parâmetro diâmetro do maior broto, conforme análise de variância não houve influência com os substratos propostos, da mesma forma se observou um crescimento das médias em respostas ao aumento na concentração do CDB nos tratamentos. Para a variável comprimento da maior raiz, observaram-se os substratos com 40,60 e $80 \%$ de CDB destacando as melhores médias, um possível motivo é o fato da porcentagem de porosidade que mostrou ser crescente nos referidos tratamentos (Figura 2).
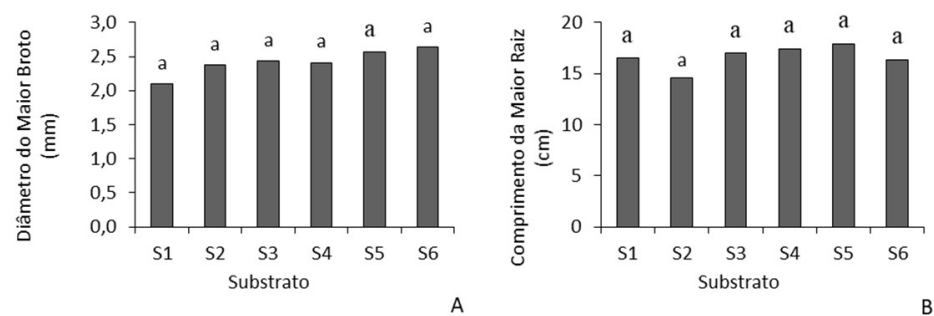

Figura 2: Diâmetro do maior broto (A) e comprimento da maior raiz (B) de mudas de amora preta com substrato a base de caule decomposto de babaçu acrescido de solo.

Com relação à área foliar (Figura 3), a proporção contendo 100\% de caule decomposto de babaçu, demonstrou eficiência para o desenvolvimento foliar. Para o volume radicular, embora nos resultados o S1 tenha apresentado a melhor média $\left(2,75 \mathrm{~cm}^{3}\right)$. Entretanto, o substrato S5 (80\% CDB) promoveu média significativamente semelhante de $2,38 \mathrm{~cm}^{3}$.
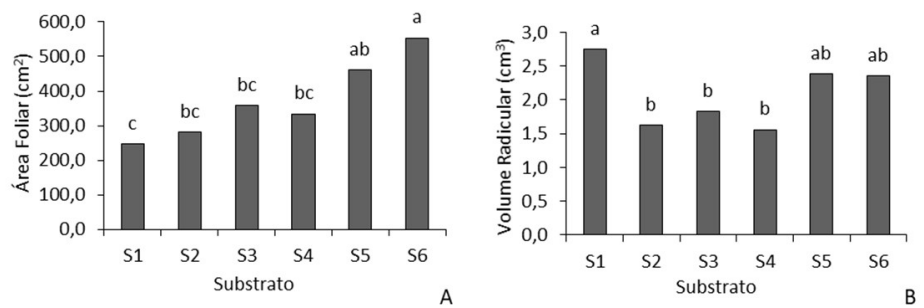

Figura 3: Área foliar (A) e volume radicular (B) de mudas de amora preta com substrato a base de caule decomposto de babaçu acrescido de solo.

As estacas de amora preta cultivadas com 100\% de CDB apresentaram os maiores acúmulos de massa fresca da raiz, a composição dos substratos com 100\%, $80 \%$ e $60 \%$ respectivamente, influenciaram estatisticamente $(p<0,05)$ essa variável, proporcionando maior desenvolvimento em relação aos demais tratamentos (Figura 4).
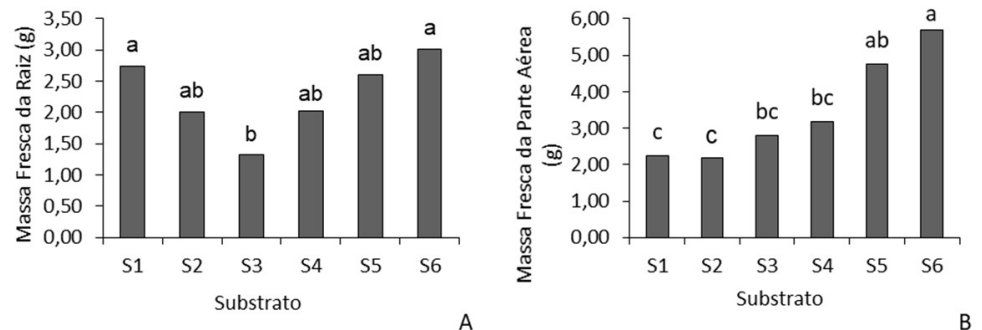

Figura 4: Massa fresca da raiz e Massa fresca da parte aérea de mudas de amora preta com substrato a base de caule decomposto de babaçu (CDB) acrescido de solo.

Para a massa fresca da parte aérea, foi a mesma tendência de desenvolvimento da massa fresca da raiz, possivelmente a composição do substrato, associado às características físicas de porosidade e 
densidade foram determinantes para destacar a proporção de 100\% de CDB para formação da massa fresca da parte aérea. Os materiais utilizados para compor substratos na propagação de mudas por estaquia têm como principais funções dar suporte as estacas durante o processo de enraizamento, com umidade e aeração em proporções adequadas (MENDES, 2017) Podendo influenciar tanto o percentual de enraizamento quanto a qualidade do sistema radicular das mudas (FRANZON et al., 2010).

O que ficou evidente com uso dos substratos compostos por maiores concentrações de caule decomposto de babaçu, se mostraram mais favoráveis para as variáveis estudadas. As concentrações de teores totais de nutrientes observados na análise química, bem como, as características físicas do substrato, devem ter proporcionado o desenvolvimento. Segundo VIEIRA e WEBER (2016) a adição de um resíduo orgânico ao substrato além de propiciar a disponibilização de nutrientes de forma adequada para as plantas, favorece também as propriedades físicas do substrato, sendo capaz de reter umidade e manter a adequada porosidade e densidade do solo para a produção de mudas de boa qualidade.

Os nutrientes influenciam diretamente no crescimento e desenvolvimentos vegetal, ocorrendo multiplicação, diferenciação celular e alongamento em resposta ao aporte nutricional do substrato (Pes e Arenhardt, 2015). Diferente do observado neste trabalho, Sousa et al. (2020) perceberam uma redução do número de folhas utilizando $100 \%$ de CDB, avaliando a produção de chrysanthemum leucanthemum com caule decomposto de babaçu.

Analisando as características do substrato (Tabela 1), com exceção do S1(0\% CDB) que apresentou $3,08 \mathrm{~g} \mathrm{~kg}^{-1}$ de $\mathrm{N}$, verificou-se valores crescente desse nutriente, o que possivelmente tenha influenciado no desenvolvimento das variáveis, devido este nutriente estar relacionado ao crescimento vegetativo. Pode-se ainda perceber elevado teor de nitrogênio nos três substratos com maiores proporções de CDB. E isso pode ter proporcionado o melhor desenvolvimento da raiz. Picolotto et al. (2015), avaliando o enraizamento de estacas de amoreira-preta em função da adubação nitrogenada, reforçam a hipótese da relação do nitrogênio com a formação de raízes em estacas de amoreira-preta.

As maiores concentrações de $\mathrm{N}, \mathrm{P}, \mathrm{Ca}$ e $\mathrm{Mg}$ foram registradas no substrato com $100 \%$ CDB, nutrientes estes, envolvidos na fotossíntese, respiração e indução do crescimento foliar, o que justifica o bom desenvolvimento da área foliar. Esta variável é influencia diretamente sobre o incremento da atividade fotossintética. Uma vez que quanto maior a área foliar, maior a capacidade de a planta produzir fotoassimilados, favorecendo o crescimento e desenvolvimento das plantas (ARAÚJO, 2015).

As concentrações de fosforo e cálcio, $555 \mathrm{mg} \mathrm{kg}^{-1}$ e $22,50 \mathrm{cmol}_{\mathrm{c}} \mathrm{kg}^{-1}$ respectivamente, observadas na análise química do substrato S1, podem ter sido responsáveis pela promoção do volume radicular em função destes nutrientes estar diretamente envolvidos no desenvolvimento e crescimento vegetativo.

Entretanto, resultado obtido com o S5 é próximo do encontrado por Santana et al. (2019), que avaliaram mudas de ipê roxo utilizando o caule decomposto de babaçu, estas apresentaram volume radicular de 2,43 $\mathrm{cm}^{3}$. Influenciado possivelmente pelo teor nutricional e ação condicionante do material orgânico utilizado para compor os substratos. Pois, segundo Malavolta e Romero (1975) esse efeito físico proporcionado pelos substratos orgânicos são fundamentais para a manutenção da disponibilidade do 
oxigênio para as raízes. E a aeração eficiente é um fator que influenciou positivamente no desenvolvimento radicular (Araujo et al., 2020).

Já os resultados de biomassa fresca diferiram do reportado por Sousa et al. (2020), que avaliando a produção de chrysanthemum leucanthemum perceberam uma melhor média utilizando $40 \%$ CDB+60\% solo para essa variável. Por outro lado, cabe ressaltar que a elevação da área foliar correlaciona-se ao bom desenvolvimento da área foliar nos substratos com maior proporção de $C D B$, uma vez que esta proporciona maior potencial fotossintético as plantas.

\section{CONCLUSÃO}

A composição dos substratos com uso caule decomposto de babaçu combinado com solo proporcionaram bom desempenho das principais variáveis. Sendo os substratos que contêm as maiores concentrações do resíduo vegetal, de forma geral, se mostraram eficientes na produção de mudas de amora.

\section{REFERÊNCIAS}

ANTUNES, L. E. C.; TREVISAN, R.; GONÇALVES, E. D. Propagação, plantio e tratos culturais. In: ANTUNES, L. E. C.; RESEIRA, M. C. B.. Aspectos técnicos da cultura da amora-preta. Pelotas: Embrapa Clima Temperado, 2004.

BICCA, M. L.; SILVA, J. P.; DIAS, C. S.; LOY, F. S.; CRUZ, J. G.; SILVA, F. L.. Substratos e ácido indolbutírico no enraizamento de miniestacas de amoreira-preta 'Xavante'coletadas em duas épocas do ano. Research, Society and Development, v.9, n.12, 2020. DOI: http://doi.org/10.33448/rsd-v9i12.10834

LONE, A. B.; UNEMOTO, L. K.; YAMAMOTO, L. Y.; COSTA, L.; SCHNITZER, J. A.; SATO, A. J.; RICCE, W. S.; ASSIS, A. M.; ROBERTO, S. R.. Enraizamento de estacas de azaleia (Rhododendron simsii Planch.) no outono em AIB e diferentes substratos. Ciência Rural, v.40, n.8, p.1720-1725, 2010. DOI: http://doi.org/10.1590/S010384782010000800008

PASSOS, M. L. V.; ZAMBRZYCKI, G. C.; PEREIRA, R. S.. Balanço hídrico e classificação climática para uma determinada região de Chapadinha-MA. Revista Brasileira de Agricultura Irrigada, v.10, n.4, p.758-766, 2016. DOI: https://doi.org/10.7127/RBAI.V10N400402

PES, L. Z.; ARENHARDT, M. H.. Solos. Santa Maria: UFSM, 2015.

PICOLOTTO, L.; VIGNOLO, G. K.; PEREIRA, I. S.; GONCALVES, M. A.; ANTUNES, L. E. C.. Enraizamento de estacas de amoreira-preta em função da adubação nitrogenada na planta matriz. Revista Ceres, v.62, n.3, p.294-300, 2015. DOI: https://doi.org/10.1590/0034-737X201562030009

SÁ, F. V. S.; BRITO, M. E. B.; MELO, A. S.; ANTÔNIO NETO, P.; FERNANDES, P. D.; FERREIRA, I. B.. Produção de mudas de mamoeiro irrigadas com água salina. Revista Brasileira de Engenharia Agrícola e Ambiental, v.17, n.10, p.1047-1054, 2013. DOI: https://doi.org/10.1590/S1415$\underline{43662013001000004}$
SANTANA, M. S.; ALMEIDA, A. P. S.; PONTES, S. F.; COSTA, C. A. A.; OLIVEIRA, A. R. F.; SILVA-MATOS, R. R. S.. Produção de mudas de ipê roxo em substratos a base de caule decomposto de babaçu. Agropecuária científica no semiárido, v.15, n.4, p.275-280, 2019. DOI: http://dx.doi.org/10.30969/acsa.v15i4.1097

SCHIEHL, M.; FRANÇA, T. O.; BIASI, L. A.. Adequação de protocolo para cultivo in vitro de amoreira-preta (Rubus sp)'Xingu'. Journal of Biotechnology and Biodiversity, v.8, n.2, p.79-87, 2020. DOI:

https://doi.org/10.20873/jbb.uft.cemaf.v8n2.schieh

SEVERINO, L. S.; LIMA, R. L. S.; BELTRÃO, N. M.. Composição química de onze materiais orgânicos utilizados em substratos para produção de mudas. Campina Grande: Embrapa Algodão (Comunicado Técnico), 2006. SILVA, F. A. S.; AZEVEDO, C. A. V.. The Assistat Software Version 7.7 and its use in the analysis of experimental data. African Journal of Agricultural Research, v.11, n.39, p.37333740, 2016.

SOUSA, G. S.; OLIVEIRA, P. S. T.; MELO, G. S.; AZEVEDO, G. A.; MENESES, K. C.; REIS, I. S.; AGUIAR, F. I. S.; SILVA-MATOS, R. R. S.. Produção de mudas de Chrysanthemum leucanthemum em substratos a base de caule decomposto de babaçu. Brazilian Journal of Development, v.6, n.6, p.40665-40675, 2020. DOI: http://doi.org/10.34117/bjdv6

MAGGIONI, M. S.; ROSA, C. B. C. J.; ROSA JUNIOR, E. J.; SILVA, E. F.; ROSA, Y. B. C. J.; SCALON, S. P. Q.; VASCONCELOS, A. A.. Development of basil seedlings (Ocimum basilicum L.) in different density and type of substrates and trays. Revista Brasileira de Plantas Medicinais, v.16, n.1, p.10-17, 2014. DOI: http://doi.org/10.1590/ S1516-05722014000100002

COELHO, J. L. S.; SILVA, R. M.; BAIMA, W. D. S.; GONSALVES, H. R. O.; NETO, F. C. S.; AGUIAR, A. V. M.. Diferentes substratos na produção de mudas de pimentão. 
Agropecuária Científica no Semiárido, v.9, n.2, p.1-4, 2013. DOI: http://dx.doi.org/10.30969/acsa.v9i2.343

ARAÚJO, E. F.. Reuso da água residuária da suinocultura na produção de mudas de essências florestais em substratos regionais. Dissertação (Mestrado em Agronomia) Universidade Federal do Piauí, Bom Jesus, 2015.

VIEIRA, C. R.; WEBER, O. L. S.. Produção de mudas de eucalipto em diferentes composições de substratos. Revista de estudos ambientais, v.18, n.2, p.25-34, 2016. DOI: http://dx.doi.org/10.7867/1983-1501.2016v18n2p25-34

MENDES, N. V. B.. Propagação vegetativa de umbucajazeira. Dissertação (Mestrado em Agronomia-Fitotecnia) Universidade Federal do Ceará, 2017.

FRANZON, R. C.; CARPENEDO, S.; SILVA, J. C. S.. Produção de mudas: principais técnicas utilizadas na propagação de fruteiras. Planaltina: Embrapa Cerrados, 2010.
LE BELLEC, F.; VAILLANT, F.; IMBERT, E.. Pitahaya ( Hylocereus spp.): a new fruit crop, a market with a future. Fruits, v.61, n.4, p.237-250, 2006. DOI: http://dx.doi.org/10.1051/2006021

FARIAS, W. C.; OLIVEIRA, L. L. P.; OLIVEIRA, T. A.; DANTAS, L. L. G. R.; SILVA, T. A. G.. Caracterização física de substratos alternativos para a produção de mudas. Agropecuária Científica no Semiárido, v.8, n.3, p.1-6, 2012. DOI: http://dx.doi.org/10.30969/acsa.v8i3.187

MALAVOLTA, E.; ROMER, J. P.. Manual de adubação. 2 ed. São Paulo: ANDA, 1975.

ARAÚJO, M. B. F.; GARRETO, V. C.; SILVA, A. S.; OLIVEIRA, A. R. F.; SILVA VIANA, J.; MACHADO, F. G. A.; OLIVEIRA, P. S. T.; CONCEIÇÃO, A. O.; SOUSA, V. A.; SILVA-MATOS, R. R. S.. Produção de mudas de amoreira com casca de arroz in natura. Research, Society and Development, v.9, n.7, 2020. DOI: https://doi.org/10.33448/rsd-v9i7.5195

A CBPC - Companhia Brasileira de Produção Científica (CNPJ: 11.221.422/0001-03) detém os direitos materiais desta publicação. Os direitos referem-se à publicação do trabalho em qualquer parte do mundo, incluindo os direitos às renovações, expansões e disseminações da contribuição, bem como outros direitos subsidiários. Todos os trabalhos publicados eletronicamente poderão posteriormente ser publicados em coletâneas impressas sob coordenação da Sustenere Publishing, da Companhia Brasileira de Produção Científica e seus parceiros autorizados. Os (as) autores (as) preservam os direitos autorais, mas não têm permissão para a publicação da contribuição em outro meio, impresso ou digital, em português ou em tradução. 